\title{
Pair Phase Fluctuations and the Pseudogap
}

\author{
T. Eckl, ${ }^{1}$ D. J. Scalapino, ${ }^{2}$ E. Arrigoni, ${ }^{1}$ and W. Hanke ${ }^{1}$ \\ ${ }^{1}$ Institut für Theoretische Physik und Astrophysik, \\ Universität Würzburg, Am Hubland, D-97074 Würzburg, Germany \\ ${ }^{2}$ Department of Physics, University of California, Santa Barbara, CA 93106-9530 USA
}

(Dated: November 6, 2018)

\begin{abstract}
The single-particle density of states and the tunneling conductance are studied for a twodimensional BCS-like Hamiltonian with a $d_{x^{2}-y^{2}}$-gap and phase fluctuations. The latter are treated by a classical Monte Carlo simulation of an $X Y$ model. Comparison of our results with recent scanning tunneling spectra of Bi-based high- $T_{c}$ cuprates supports the idea that the pseudogap behavior observed in these experiments can be understood as arising from phase fluctuations of a $d_{x^{2}-y^{2}}$ pairing gap whose amplitude forms on an energy scale set by $T_{c}^{M F}$ well above the actual superconducting transition.
\end{abstract}

PACS numbers: 71.10.Fd, 71.27.+a, 74.25.Jb, 74.72.Hs

Intensive research has focused on the pseudogap regime, which is observed in the high- $T_{c}$ cuprates below a characteristic temperature that is higher than the transition temperature $T_{c}$. It occurs in a number of different experiments as a suppression of low-frequency spectral weight [1, 2, 3, , 5, 6, 6, 8. This striking pseudogap behavior initiated a variety of proposals as to its origin 19, 10, 11, 12, 13, 14, 15, 16, since the answer to this question may be a key ingredient for the understanding of high- $T_{c}$ superconductivity. At present, there is no agreement as to which of these proposals is correct. In part, this reflects the possibility that there may be different pseudogap phenomena operating in different temperature and doping regimes. In part, this is because of the difficulty in determining the experimental consequences of the various theoretical proposals. In this paper, we focus on the pseudogap phenomena observed in scanning tunneling spectroscopy measurements [6, 7] on $\mathrm{Bi}_{2} \mathrm{Sr}_{2} \mathrm{CaCu}_{2} \mathrm{O}_{8+\delta}$ (Bi2212) and $\mathrm{Bi}_{2} \mathrm{Sr}_{2} \mathrm{CuO} \mathrm{O}_{6+\delta}$ (Bi2201). We provide a detailed numerical solution of a minimal model which, however, contains the key ideas of the cuprate phase fluctuation scenario: that is, we explore the notion that the pseudogap observed in these experiments arises from phase fluctuations of the gap [6, 6, 12, 13, 14, 15]. In this scenario, below a mean field temperature scale $T_{c}^{M F}$, a $d_{x^{2}-y^{2} \text {-wave }}$ gap amplitude is assumed to develop. However, the superconducting transition is suppressed to a considerably lower temperature $T_{c}$ by phase fluctuations 12 . In the intermediate temperature regime between $T_{c}^{M F}$ and $T_{c}$, the phase fluctuations of the gap give rise to pseudogap phenomena.

We will study as a model for phase fluctuations a twodimensional BCS Hamiltonian

$H=-t \sum_{\langle i j\rangle, \sigma}\left(c_{i \sigma}^{\dagger} c_{j \sigma}+c_{j \sigma}^{\dagger} c_{i \sigma}\right)-\frac{1}{4} \sum_{i \delta}\left(\Delta_{i \delta}\left\langle\Delta_{i \delta}^{\dagger}\right\rangle+\Delta_{i \delta}^{\dagger}\left\langle\Delta_{i \delta}\right\rangle\right)$

where $c_{i \sigma}^{\dagger}$ creates an electron of spin $\sigma$ on the $i^{\text {th }}$ site and $t$ denotes an effective nearest-neighbor hopping. The $\langle i j\rangle$ sum is over nearest-neighbor sites of a $2 \mathrm{D}$ square lattice, and in the second term $\delta$ connects $i$ to its nearestneighbor sites. In Eq. (11) one could, of course, add a next-near-neighbor hopping $t^{\prime}$ and a chemical potential term. Here, for simplicity and to refrain from further approximations, we have set $t^{\prime}$ and the chemical potential equal to zero [17]. We will assume that below a mean field temperature $T_{c}^{M F}$, a $d_{x^{2}-y^{2} \text {-gap amplitude forms }}$ with $\Delta \sim 2 T_{c}^{M F}$. The detailed temperature dependence of $\Delta$ is not central, as we are not interested in the region around $T_{c}^{M F}$ where the pseudogap closes. The important point for our calculations is simply that a $d_{x^{2}-y^{2} \text {-gap }}$ amplitude of order $2 T_{c}^{M F}$ in magnitude forms as $T$ drops below $T_{c}^{M F}$ so that

$$
\left\langle\Delta_{i \delta}^{\dagger}\right\rangle=\frac{1}{\sqrt{2}}\left\langle c_{i \uparrow}^{\dagger} c_{i+\delta \downarrow}^{\dagger}-c_{i \downarrow}^{\dagger} c_{i+\delta \uparrow}^{\dagger}\right\rangle=\Delta e^{i \Phi_{i \delta}},
$$

with

$$
\Phi_{i \delta}= \begin{cases}\left(\varphi_{i}+\varphi_{i+\delta}\right) / 2 & \text { for } \delta \text { in x-direction } \\ \left(\varphi_{i}+\varphi_{i+\delta}\right) / 2+\pi & \text { for } \delta \text { in y-direction }\end{cases}
$$

We then determine the fluctuating phases from a Monte Carlo calculation using an effective 2D $X Y$-free energy

$$
F\left[\varphi_{i}\right]=-E_{1} \sum_{\langle i j\rangle} \cos \left(\varphi_{i}-\varphi_{j}\right)
$$

with $E_{1}$ adjusted to set the Kosterlitz-Thouless 18 transition temperature $T_{K T}$ equal to some fraction of $T_{c}^{M F}$. Specifically, for the present calculation we will set $T_{K T} \simeq T_{c}^{M F} / 5$. Here, we have the recent scanning tunneling results [7] for $\mathrm{Bi}_{2} \mathrm{Sr}_{2} \mathrm{CuO}_{6+\delta}$ in mind, where $T_{c} \simeq 10 \mathrm{~K}$ and the pseudogap regime extends to 50 or $60 \mathrm{~K}$, which we take as $T_{c}^{M F}$.

In principle, the $X Y$ action, which determines the fluc, tuations of the phases, arises from integrating out the shorter wavelength fermion degrees of freedom including those responsible for the local pair amplitude and the internal $d_{x^{2}-y^{2}}$ structure of the pairs. In general this leads 
to a $\tau$-dependent quantum action as well as a coupling energy $E_{1}$, whose temperature dependence is determined by the many-body interactions of the microscopic system. There have been various discussions regarding the regime over which a classical action is appropriate for the cuprates [19, 20, 21]. Here, however, we will proceed phenomenologically using the classical action, Eq. (1), and neglecting the temperature dependence of $E_{1}$. Furthermore, we will use the 2D form of Eq. (何). One knows that for the layered cuprates there is a crossover from $2 \mathrm{D}$ to 3D $X Y$ behavior near $T_{c}$ 22. Our point of view is that away from this crossover regime, a $2 \mathrm{D}$ model is certainly suitable and on the finite size lattice that we will study, the system becomes effectively ordered as $T$ approaches $T_{K T}$ and the correlation length exceeds the lattice size. So $E_{1}$ will simply be used to set $T_{K T} \equiv T_{c}$. A crucial physical point that will be taken into account in our analysis is that the basic length scale of the $\varphi$-field is larger than the Cooper-pair size $\xi_{0}$. Thus, although this is a clearly simplified model, we believe that its solution provides useful insight into the experimental consequences of the phase fluctuation pseudogap scenario. It is the central aim of this paper to verify this by comparison with the STM experiments and reproduction of some of their characteristic and salient features.

The calculation of the density of states for an $L \times L$ periodic lattice now proceeds as follows 23, 24. A set of phases $\left\{\varphi_{i}\right\}$ is generated by a Monte Carlo (MC) importance sampling procedure, in which the probability of a given configuration is proportional to $\exp \left(-F\left[\varphi_{i}\right] / T\right)$ with $F$ given by Eq. (4). With $\left\{\varphi_{i}\right\}$ given, the Hamiltonian of Eq. (11) is diagonalized and the single particle density of states $N\left(\omega, T,\left\{\varphi_{i}\right\}\right)$ is calculated. Further MC $\left\{\varphi_{i}\right\}$ configurations are generated and an average density of states $N(\omega, T)=\left\langle N\left(\omega, T,\left\{\varphi_{i}\right\}\right)\right\rangle$ at a given temperature is determined.

As noted above, our point of view is that the $X Y$ action, used in the MC simulations, in principle arises from integrating out the shorter wavelength fermion degrees of freedom up to the scale of the Cooper-pair size, so that only the center of mass pair phase fluctuations are important. Thus, the scale of the lattice spacing for $F\left[\varphi_{i}\right]$ is set by the pair size coherence length $\xi_{0} \sim v_{F} / \pi \Delta_{0}$ and is of order 3 to 4 times the basic $C u$ - $C u$ lattice spacing of the fermion Hamiltonian Eq. (11). Now the computationally intensive part of the calculation is the diagonalization of $H$ and in order to get meaningful results as $T$ approaches $T_{K T}$, we found it necessary to average over a large number of Monte Carlo $\left\{\varphi_{i}\right\}$ configurations. This requires that some compromise be made with respect to the lattice size. The results, we will present, are for a $32 \times 32$ Hamiltonian lattice. However, if we were to take $\xi_{0} \sim 4$ lattice spacings, this would lead to only an $8 \times 8$ lattice for the $\varphi_{i}$ simulations. This would not allow a sufficient range for the Kosterlitz-Thouless phase coherence length to grow as $T$ approaches $T_{K T}$.
Thus, we have chosen to set $\Delta=1.0 t$ giving $\xi_{0} \sim 1$ so that the $\varphi_{i}$ simulation can be carried out on the same $L \times L$ lattice that is used for the diagonalization of $H$. The important physical point is that this procedure effectively cuts off phase fluctuations on a scale less than the Cooper-pair size, $\xi_{0}$. Thus, the phase coherence length is always larger than the Cooper-pair size when $T$ is less than $T_{c}^{M F}$. Consequently, our results differ from earlier work [25], which found that the pseudogap regime due to fluctuating phases extended only about $20 \%$ above $T_{c}$, in contrast to the Bi tunneling experiments [6, 7] and the recent Nernst-effect results [8]. In the work of Ref. [25], parameters were used which set the basic scale of the phase correlation length to be much smaller than $\xi_{0}$ and, therefore, the phase correlation length exceeded $\xi_{0}$ only in a narrow temperature region set by a fraction of $T_{K T}$. We believe that this is not the correct phenomenology.

Results for $N(\omega, T)$ are shown in Fig. 1. For each tem-

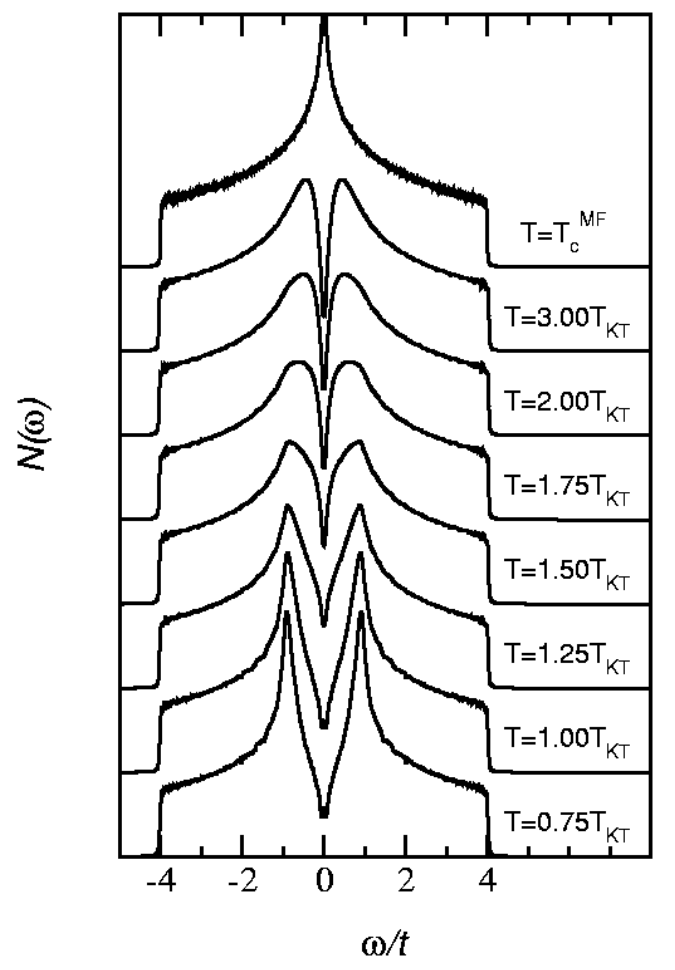

FIG. 1: Single particle density of states $N(\omega)$ for different temperatures $T$ for a $32 \times 32$ lattice with $\Delta=1.0 t$ and $T_{K T}=$ $0.1 t$. A pseudogap appears below $T_{c}^{M F} \simeq 0.5 t$ and coherence peaks develop as $T$ approaches $T_{K T}$.

perature we have generated up to 25,000 independent MC $\left\{\varphi_{i}\right\}$ configurations, diagonalized $H$ for each of these configurations, and computed $\left\langle N\left(\omega, T,\left\{\varphi_{i}\right\}\right)\right\rangle$. In these calculations, as discussed above, we have set $\Delta=1.0 t$ corresponding to $T_{c}^{M F} \simeq 0.5 t$ and selected $E_{1}$ so that $T_{K T}=0.1 t$ [26]. In order to reduce finite-size effects, we employ a very effective scheme recently suggested by F. F. Assaad [27]. 
For $T>T_{c}^{M F}$, the gap amplitude vanishes and the density of states exhibits the usual Van Hove peak at $\omega=0$. For $T<T_{c}^{M F}$, the presence of a finite gap amplitude gives rise to a pseudogap whose size is set by $2 \Delta$. Then, as $T$ approaches $T_{K T}$ and the $X Y$ phase correlation length rapidly increases, coherence peaks evolve, the separation of which is determined by $2 \Delta$. An important point is that the scale in temperature over which the evolution of the coherence peaks occurs, is set by some fraction of $T_{K T}$ which means that it appears suddenly on a scale set by $T_{c}^{M F}$.

An effective correlation length $\xi(T)$, extracted by fitting an exponential form to the correlation function

$$
C(\ell)=\left\langle e^{-i \varphi_{i+\ell}} e^{i \varphi_{i}}\right\rangle
$$

is plotted versus $T$ in Fig. 2 for our $32 \times 32$ lattice. The

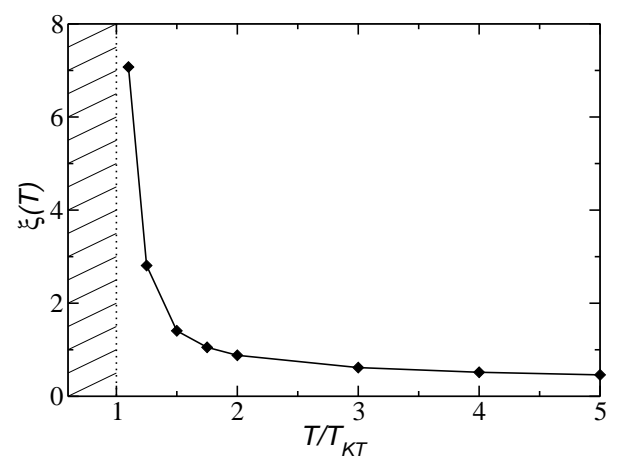

FIG. 2: The effective correlation length $\xi(T)$ versus $T / T_{K T}$ for the $32 \times 32$ lattice. Here $T_{c}^{M F} / T_{K T} \simeq 5$ so that the pseudogap regime which extends from $T / T_{K T} \simeq 1.5$ to 5 is large compared to the superconducting region which extends from 0 to $T / T_{K T}=1$. The pronounced increase of $\xi(T)$ occurs over a narrow temperature region, on a scale set by $T_{c}^{M F}$, as $T_{K T}$ is approached.

rapid onset of $\xi(T)$ as $T_{K T}$ is approached is clearly seen. It is this sudden increase of $\xi(T)$ that is responsible for the appearance of the coherence peaks as $T$ approaches $T_{K T}$. This effect is further enhanced by the $2 D$ to $3 D$ crossover that occurs in the actual materials.

In order to compare these results for $N(\omega, T)$ with scanning tunneling spectra $d I / d V$, we have calculated $d I(V, T) / d V$ using the standard quasi-particle expression for the tunneling current,

$$
\frac{d I(V, T)}{d V} \propto \int N(\omega) \frac{\partial f(\omega-V)}{\partial V} d \omega .
$$

Here, $f(\omega)=(\exp (\omega / T)+1)^{-1}$ is the usual Fermi factor. Results for $d I(V, T) / d V$ are displayed in Fig. 3. The effect of the Fermi factors is to provide a thermal smoothing of the quasi-particle density of states over a region of order $2 T$. This becomes significant at the higher temperatures and the prominent pseudogap dependence of

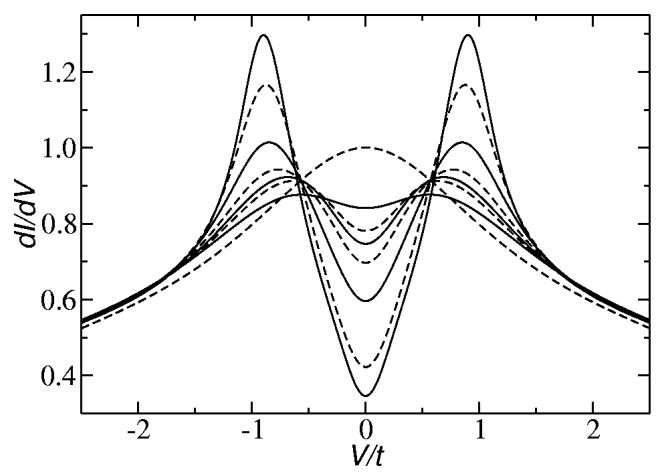

FIG. 3: Tunneling conductance, $\frac{d I}{d V}$, normalized to its value at $T_{c}^{M F}$ and $V=0$, for different temperatures. Solid curves are for $T=\{0.75,1.25,1.75,3.00\} T_{K T}$, dashed curves for $T=\{1.00,1.50,2.00\} T_{K T}$ and $T_{c}^{M F}\left(\left.\frac{d I}{d V}\right|_{V=0}\right.$ is increasing with $T)$.

$N(\omega, T)$ seen in Fig. 11 is smoothed out in $d I / d V$. In Fig. 田, $d I / d V$ results are shown as solid curves for $T=$ $0.75 T_{K T}$ (Fig. 四a), $T=T_{K T}$ (Fig. 四) and $T=2 T_{K T}$ (Fig. Ac). The dashed curve is for $T=T_{c}^{M F} \simeq 5 T_{K T}$. One sees that the size of the pseudogap scales with the spacing between the coherence peaks and evolves continuously out of the superconducting state. The pseudogap persists over a large temperature range measured in units of $T_{K T}$, becoming smoothed out by the thermal effects as $T$ approaches $T_{c}^{M F}$ and vanishing above $T_{c}^{M F}$.

Our numerical results for $d I(V, T) / d V$ are similar to recent scanning tunneling measurements of Bi2212 and Bi2201 [6, 7]. Also in these experiments the superconducting gap for $T<T_{K T}$ evolves continuously into the pseudogap regime, which extends up to $T=T_{c}^{M F}$. The coherence peaks appear suddenly as $T_{K T}$ is approached. At higher temperatures, the pseudogap fills in rather than closing and the temperature range associated with the pseudogap regime can be large compared with the size of the superconducting regime.

Summarizing, in order to develop a more quantitative understanding for the role of phase fluctuations, we have provided a numerical solution of a simplified model which, nevertheless, contains the key ideas of the cuprate phase fluctuation pseudogap scenario. Here the center of mass pair-phase fluctuations of a BCS $d$-wave model were determined from a classical 2D $X Y$ action by means of a Monte Carlo simulation. The resulting tunneling conductance $(d I / d V)$ reproduces characteristic and salient features of recent STM studies of Bi2212 and Bi2201 suggesting that the pseudogap behavior observed in these experiments arises from phase fluctuations of the $d_{x 2-y 2^{-}}$ pairing gap.

We would like to acknowledge useful discussions with S. A. Kivelson and A. Paramekanti. This work was supported by the DFG under Grant No. Ha 1537/16-2 and AR 324/3-1, by the Bavaria California Technology Cen- 

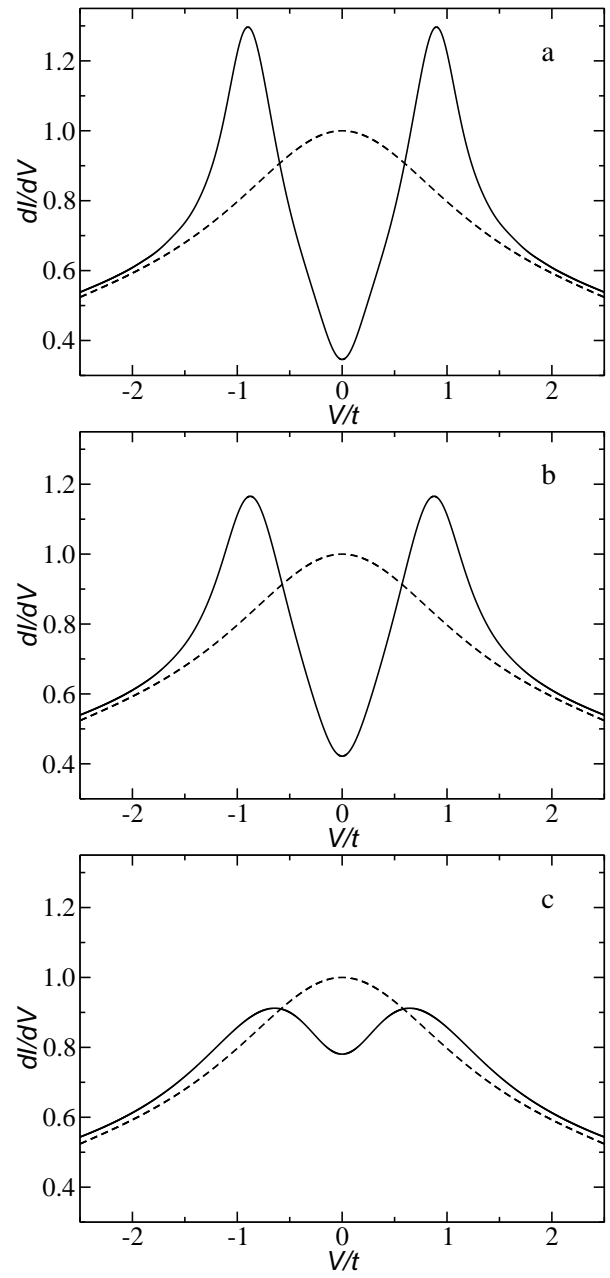

FIG. 4: Temperature dependence of $\frac{d I}{d V}$ normalized to its value at $T_{c}^{M F}$ and $V=0$. The solid curves are for $T=$ $0.75 T_{K T}$ (a), $T=T_{K T}$ (b) and $T=2 T_{K T}$ (c). The dashed curve in all three figures is for $T=T_{c}^{M F} \simeq 5 T_{K T}$.

ter $(\mathrm{BaCaTeC})$, the superconducting project KONWHIR OOPCV and by the U. S. Department of Energy under Grant No. DE-FG03-85ER45197. The calculations were carried out at the high-performance computing centers HLRS (Stuttgart) and LRZ (München).

[1] A. G. Loeser, Z. X. Shen, D. S. Dessau, D. S. Marshall, C. H. Park, P. Fournier, and A. Kapitulnik, Science 273, 325 (1996).

[2] H. Ding, T. Yokoya, J. C. Campuzano, T. Takahashi, M. Randeria, M. R. Norman, T. Mochiku, K. Kadowaki, and J. Giapintzakis, Nature (London) 382, 51 (1996); H. Ding, M. R. Norman, T. Yokoya, T. Takeuchi, M. Randeria, J. C. Campuzano, T. Takahashi, T. Mochiku, and K. Kadowaki, Phys. Rev. Lett. 78, 2628 (1997).

[3] J. W. Loram, K. A. Mirza, J. R. Cooper, and
W. Y. Liang, Phys. Rev. Lett. 71, 1740 (1993).

[4] M. Takigawa, A. P. Reyes, P. C. Hammel, J. D. Thompson, R. H. Heffner, Z. Fisk, and K. C. Ott, Phys. Rev. B 43, 247 (1991); H. Alloul, A. Mahajan, H. Casalta, and O. Klein, Phys. Rev. Lett. 70, 1171 (1993).

[5] J. Orenstein, G. A. Thomas, A. J. Millis, S. L. Cooper, D. H. Rapkine, T. Timusk, L. F. Schneemeyer, and J. V. Waszczak, Phys. Rev. B 42, 6342 (1990).

[6] Ch. Renner, B. Revaz, J.-Y. Genoud, K. Kadowaki, and Ø. Fischer, Phys. Rev. Lett. 80, 149 (1998); N. Miyakawa, J. F. Zasadzinski, L. Ozyuzer, P. Guptasarma, D. G. Hinks, C. Kendziora, and K. E. Gray, Phys. Rev. Lett. 83, 1018 (1999).

[7] M. Kugler, Ø. Fischer, Ch. Renner, S. Ono, and Y. Ando, Phys. Rev. Lett. 86, 4911 (2001).

[8] Y. Wang, N. P. Ong, Z. A. Xu, T. Kakeshita, S. Uchida, D. A. Bonn, R. Liang, and W. N. Hardy, Phys. Rev. Lett. 88, 257003 (2002)

[9] S. Chakravarty, R. B. Laughlin, D. K. Morr, and C. Nayak, Phys. Rev. B 63, 94503 (2001).

[10] J. R. Schrieffer and A. P. Kampf, J. Phys. Chem. Solids 56, 1673 (1995).

[11] D. Pines, Z. Phys. B 103, 129 (1997); A. V. Chubukov and J. Schmalian, Phys. Rev. B 57, R11085 (1998).

[12] V. J. Emery and S. A. Kivelson, Nature (London) 374, 434 (1995).

[13] M. Randeria, N. Trivedi, A. Moreo, and R. T. Scalettar, Phys. Rev. Lett. 69, 2001 (1992).

[14] M. Franz and A. J. Millis, Phys. Rev. B 58, 14572 (1998).

[15] H.-J. Kwon and A. T. Dorsey, Phys. Rev. B 59, 6438 (1999).

[16] B. Kyung, S. Allen and A.-M. S. Tremblay, Phys. Rev. B 64, 75116 (2001).

[17] We have checked to some extent that our results do not depend appreciably on $t^{\prime}$ or $\mu$.

[18] J. M. Kosterlitz and D. J. Thouless, J. Phys. C 6, 1181 (1973).

[19] V. J. Emery and S. A. Kivelson, Phys. Rev. Lett. 74, 3253 (1995).

[20] A. Paramekanti, M. Randeria, T. V. Ramakrishnan, and S. S. Mandal, Phys. Rev. B 62, 6786 (2000).

[21] L. Benfatto, S. Caprara, C. Castellani, A. Paramekanti, and M. Randeria, Phys. Rev. B 63, 174513 (2001).

[22] E. W. Carlson, S. A. Kivelson, V. J. Emery, and E. Manousakis, Phys. Rev. Lett. 83, 612 (1999) and references therein.

[23] A more detailed discussion of this static field Monte Carlo approximation for the pair-field is given in T. Eckl et al. (to be published); see also N. E. Bickers and D. J. Scalapino, cond-mat/0010480 (unpublished), and P. Monthoux and D. J. Scalapino, Phys. Rev. B 65, 235104 (2002).

[24] E. Dagotto, S. Yunoki, A. L. Malvezzi, A. Moreo, J. Hu, S. Capponi, D. Poilblanc, N. Furukawa, Phys. Rev. B 58, 6414 (1998) have used Monte Carlo simulations to study a model of classical spins interacting with electrons. The effects of phase fluctuations have been addressed within a quasi-classical approximation by H. Monien, J. Low Temp. Phys. 126, 1123 (2002).

[25] P. E. Lammert and D. S. Rokhsar, cond-mat/0108146 (unpublished).

[26] $T_{K T} \simeq 0.89 E_{1}$, see, for example, J. F. Fernández, M. F. Ferreira, and J. Stankiewicz, Phys. Rev. B 34, 292 (1986). 
[27] F. F. Assaad, Phys. Rev. B 65, 115104 (2002). 